\title{
International Prognostic Scoring System (IPSS) for Myelodysplastic Syndromes
}

National Cancer Institute

\section{Source}

National Cancer Institute. International Prognostic Scoring System (IPSS) for

Myelodysplastic Syndromes. NCI Thesaurus. Code C84350.

A system for scoring myelodysplasia syndrome progression risk, based on a combination of: percentage of blast cells in the bone marrow, the presence and severity of specific cytogenetic abnormalities, and the number of cytopenias in the peripheral blood. 